\title{
PCTR1 ameliorates lipopolysaccharide-induced acute inflammation and multiple organ damage via regulation of linoleic acid metabolism by promoting FADS1/FASDS2/ELOV2 expression and reducing PLA2 expression
}

\author{
Yong-jian $\mathrm{Liu}^{1} \cdot \mathrm{Hui} \mathrm{Li}^{1} \cdot$ Yang Tian ${ }^{1} \cdot$ Jun $\mathrm{Han}^{1} \cdot$ Xin-yang Wang ${ }^{1} \cdot$ Xin-yu Li ${ }^{1} \cdot$ Chao Tian ${ }^{1} \cdot$ Pu-hong Zhang ${ }^{1}$. \\ Yu Hao ${ }^{1} \cdot$ Fang Gao $^{1,2} \cdot$ Sheng-wei Jin ${ }^{1}$
}

Received: 21 October 2019 / Revised: 18 February 2020 / Accepted: 18 February 2020 / Published online: 2 March 2020

(c) The Author(s), under exclusive licence to United States and Canadian Academy of Pathology 2020

\begin{abstract}
Gram-negative bacterial infection causes an excessive inflammatory response and acute organ damage or dysfunction due to its outer membrane component, lipopolysaccharide (LPS). Protectin conjugates in tissue regeneration 1 (PCTR1), an endogenous lipid mediator, exerts fundamental anti-inflammation and pro-resolution during infection. In the present study, we examined the properties of PCTR1 on the systemic inflammatory response, organic morphological damage and dysfunction, and serum metabolic biomarkers in an LPS-induced acute inflammatory mouse model. The results show that PCTR1 reduced serum inflammatory factors and ameliorated morphological damage and dysfunction of the lung, liver, kidney, and ultimately improved the survival rate of LPS-induced acute inflammation in mice. In addition, metabolomics analysis and high performance liquid chromatography-mass spectrometry revealed that LPS-stimulated serum linoleic acid (LA), arachidonic acid (AA), and prostaglandin E2 (PGE2) levels were significantly altered by PCTR1. Moreover, PCTR1 upregulated LPS-inhibited fatty acid desaturase 1 (FADS1), fatty acid desaturase 2 (FADS2), and elongase of very long chain fatty acids 2 (ELOVL2) expression, and downregulated LPS-stimulated phospholipase A2 (PLA2) expression to increase the intrahepatic content of AA. However, these effects of PCTR1 were partially abrogated by a lipoxin A4 receptor (ALX) antagonist (BOC-2). In summary, via the activation of ALX, PCTR1 promotes the conversion of LA to AA through upregulation of FADS1, FADS2, and ELOVL2 expression, and inhibits the conversion of bound AA into free AA through downregulation of PLA2 expression to decrease the serum AA and PGE2 levels.
\end{abstract}

Yu Hao

haoyu8403@163.com

$\triangle$ Fang Gao

f.gaosmith@bham.ac.uk

$\triangle$ Sheng-wei Jin

jinshengwei69@163.com

1 Department of Anaesthesia and Critical Care, the Second Affiliated Hospital and Yuying Children's Hospital of Wenzhou Medical University, 325027 Zhejiang, PR China

2 Academic Department of Anesthesia, Critical Care, Resuscitation and Pain, Heart of England NHS Foundation Trust, Bordesley Green, Birmingham, United Kingdom

\section{Introduction}

Despite advances in antimicrobial therapy and overall medical care, gram-negative bacterial infection remains a common, life-threatening event [1]. The main causative agent of gram-negative bacteria is lipopolysaccharide (LPS), a key outer membrane component, which may induce excessive inflammatory response and acute organ damage or dysfunction [1,2]. LPS promotes the activity and expression of phospholipase A2 (PLA2), while PLA2 catalyzes the hydrolyzation of bound arachidonic acid (AA), present in the phospholipids of cellular membranes, into free AA that is subsequently released into the bloodstream. Then, free AA is metabolized into pro-inflammatory cytokines, such as PGE2, thromboxane A2 (TXA2), and leukotriene B4 (LTB4), resulting in an inflammatory response $[3,4]$. 
Metabolomics-based approaches allow the identification of variations in metabolite profiles that can be used to discriminate diseases [5]. Our previous research has shown that the levels of certain metabolites were involved in energy metabolism and inflammatory processes in the cecal ligation and puncture (CLP)-induced sepsis mouse model [6]. Thus, metabolomics may be a potentially promising tool for the diagnosis of gram-negative bacterial infection and endotoxemia.

Protectin conjugates in tissue regeneration 1 (PCTR1), a newly discovered member of specialized pro-resolving mediators (SPMs), is synthesized from docosahexaenoic acid (DHA) in leukocytes, and is highly presented in lymphatic tissues [7]. Although its specific receptor is still unknown [7], PCTR1 was shown to have fundamentally anti-inflammatory properties during microbial infection in vivo [8]. Many of the current anti-inflammatory therapies target the classical inflammatory molecules and pathways involved in the initiation of the acute inflammatory response [9]. Complications and unwanted side effects have arisen from treatments that are 'resolution toxic' through disruption of the temporal orchestration and program of resolution [9]. As an endogenous mediator, PCTR1 may circumvent the hurdles faced in the clinical implementation of resolution-toxic therapies [10]. We have reported that other members of the SPMs family, such as lipoxin A4, resolvin $\mathrm{D} 1$, maresin 1, and protectin DX, reduce inflammation, attenuate neutrophil infiltration to the tissues, and protect mitochondrial function and organ function in LPS-induced acute inflammatory model [11-13]. However, it is still unclear whether PCTR1 has an important role in LPSinduced acute inflammation, organ damage and dysfunction, moreover, the underlying mechanisms are unknown.

The present study tested the hypothesis that the administration of PCTR1 could protect against inflammation, organ damage in a mouse model of LPS-induced acute inflammation. Subsequently, biomarkers of serum metabolism were identified to explore the metabolic pathways. Finally, we determined the effects of PCTR1 on metabolites and metabolic enzymes to attain a better understanding of the underlying mechanisms.

\section{Materials and methods}

\section{Materials}

PCTR1 (17-hydroxydocosa-4,7,10,12,14,19-hexaenoic acid) was obtained from Cayman Chemical Company (Ann Arbor, MI). BOC-2 (ALX antagonist) was purchased from Biomol-Enzo Life Sciences (Farmingdale, NY). Interleukin $1 \beta$ (IL-1 $\beta$ ), interleukin 6 (IL-6), interleukin 10 (IL-10), tumor necrosis factor- $\alpha$ (TNF- $\alpha)$, macrophage inflammatory protein 2 (MIP-2), myeloperoxidase (MPO), and PGE2 ELISA kits were purchased from R\&D Systems (Minneapolis, MN, USA). Serum glutamic pyruvic transaminase (ALT), serum glutamic oxaloacetic transaminase (AST), blood urea nitrogen (BUN), blood creatinine (CREA), reactive oxygen species (ROS), superoxide dismutase (SOD), and glutathioneperoxidase 4 (GPX4) kits were purchased from Jiancheng Bioengineering Institute (Nanjing, Jiangsu, China). PLA2 ELISA kits were purchased from WESTTANG BIO-TECH CO. (Shanghai, China). High performance liquid chromatography-mass spectrometry (HPLC-MS) kits for LA and AA were purchased from Dr. Ehrenstorfer (Augsburg, Germany). LPS (Escherichia coli serotype 055: B5) was obtained from Sigma (St. Louis, MO). Quantitative real-time PCR kits were purchased from TaKaRa (Japan).

\section{Animals}

C57BL/6J wild-type male mice (8-10 weeks old, 22-25 g) were purchased from Beijing Vital River Laboratory Animal Technology Co., Ltd.. Female mice were not used due to the anti-inflammatory and antimicrobial effects of estrogen and progesterone [14]. All mice were housed littermates in groups of four per cage and maintained in a specific pathogen-free room with controlled temperature $\left(22-26^{\circ} \mathrm{C}\right)$ and humidity (60-65\%) with a regular $12 \mathrm{~h}$ light and dark cycle. We conducted all animal experiments in accordance with Laboratory Animal Guidelines for Ethical Review of Animal Welfare (GB/T 35892-2018, China) and with approval from Laboratory Animal Ethics Committee of Wenzhou Medical University.

Mice were randomly allocated into one of four groups, control, LPS, LPS + PCTR1, or LPS + PCTR1 + BOC-2. In all treatment groups, the model of acute inflammation was achieved by the intraperitoneal (i.p.) injection of $10 \mathrm{mg} / \mathrm{kg}$ LPS; mice in the control group were received an equivalent volume of saline by i.p.. Mice in the LPS + PCTR1 group were received an injection of PCTR1 (50 ng/ mice) via the tail vein at $1 \mathrm{~h}$ or $6 \mathrm{~h}$ after LPS administration. Mice in the LPS + PCTR1 + BOC-2 group were received an injection of BOC-2 $(600 \mathrm{ng} / \mathrm{kg})$ via the tail vein $0.5 \mathrm{~h}$ before LPS challenge, followed by an injection of PCTR1 (50 $\mathrm{ng} /$ mice) via the tail $1 \mathrm{~h}$ after LPS challenge.

\section{Survival analysis}

PCTR1 (50 ng/mice) or an equivalent volume of saline was randomly administered to the mice via the tail vein $1 \mathrm{~h}$ after LPS $(15 \mathrm{mg} / \mathrm{kg})$ intraperitoneal injection. The survival rate was recorded every day for 7 days. 


\section{Pathological and biological studies}

At $6 \mathrm{~h}$ after LPS injection, the mice were sacrificed, and tissues samples from the lung, liver, and kidney were immediately obtained. The tissues were embedded in paraffin, stained with hematoxylin and eosin (H\&E), and analyzed by using a light microscope. Organ injury scores were determined by two independent investigators blinded to the research assignment and following the applicable criteria [10]. At $6 \mathrm{~h}$ or $11 \mathrm{~h}$ after LPS challenge, the whole blood samples of mice were obtained and then serum was collected. The serum levels of IL-1 $\beta$, IL-6, IL-10, TNF- $\alpha$, MIP-2, MPO, PGE2, ALT, AST, BUN, CREA, ROS, SOD, GPX4, and the concentrations of PLA2 in liver tissue homogenate were determined by using ELISA kits in accordance with the manufacturer's protocols. The serum levels and liver tissue contents of LA and AA were detected by HPLC-MS with procedures as previously described [15].

\section{Samples collection and extraction for metabolomics}

The serum samples of mice were collected, and then frozen rapidly in liquid nitrogen, and stored at $-80^{\circ} \mathrm{C}$ until analyzed. The extraction was performed as previously described [16]. Briefly, $50 \mu \mathrm{L}$ serum sample was placed in $1.5 \mathrm{~mL}$ microfuge tube and extracted with $200 \mu \mathrm{L}$ methanol. $5 \mu \mathrm{L}$ of 2-chlor-L-phenylalanine was added as an internal standard and the solution was vortex mixed for $30 \mathrm{~s}$. Samples were kept at $-20^{\circ} \mathrm{C}$ for $10 \mathrm{~min}$ and centrifuge for $15 \mathrm{~min}$ at $12,000 \mathrm{~g}, 4^{\circ} \mathrm{C} .180 \mu \mathrm{L}$ of supernatant was transferred into a fresh $1.5 \mathrm{~mL}$ tube, and then $30 \mu \mathrm{L}$ were taken from from each sample and pooled as a QC sample. The supernatant was dried completely in a vacuum concentrator without heating. Then, $30 \mu \mathrm{L}$ methoxyamine hydrochloride $(20 \mathrm{mg} / \mathrm{mL}$ in pyridine) was added and incubated for $30 \mathrm{~min}$ at $80^{\circ} \mathrm{C}, 40 \mu \mathrm{L}$ of BSTFA (1\% TMCS, v/v) reagent was added to the sample aliquots and incubated for $1.5 \mathrm{~h}$ at $70^{\circ} \mathrm{C}$. All samples were analyzed by using gas chromatography coupled with time-offlight mass spectrometry (GC-TOF-MS).

\section{GC-TOF-MS analysis}

GC-TOF-MS analysis was performed by using an Agilent 7890 gas chromatograph system coupled with a Pegasus HT time-of-flight mass spectrometer. The mass spectrometry data were acquired in full scan mode with a $\mathrm{m} / \mathrm{z}$ range of $50-500$ at a rate of 12.5 spectra per second after a solvent delay of $4.78 \mathrm{~min}$. Chroma TOF $4.3 \mathrm{X}$ software and the LECO-Fiehn Rtx 5 database were used for the data preprocessing and annotation. Both mass spectrum match and retention index match were considered in metabolite identification. Peaks detected in $<50 \%$ of QC samples or RSD $30 \%$ in QC samples were removed [17].

\section{Quantitative real-time PCR}

Quantitative RT-PCR was performed by using TB Green System (TaKaRa, Japan) in accordance with the manufacturer's protocol and the data were detected by the CFX96 Real-Time PCR Detection System (Bio-Rad). The expression of target genes was analyzed by using the $2^{-\Delta \Delta} \mathrm{Ct}$ method. The gene-specific primers are as follows: mFADS1-F $5^{\prime}$-ACCTGTCAGTCTTTGGCACCTC-3 ' and mFADS1-R $5{ }^{\prime}$-TCCTTGCGGAAGCAGTTAGGCT-3'; mFADS2-F 5'-TTCCTGGAGAGCCACTGGTTTG-3' and mFADS2-R 5'-GAAGAAGGACTGCTCCACATTGC-3' ; mELOVL2-F $5^{\prime}$-AGCTGGGAAGGAGGTTACAA- $3^{\prime}$ and mELOVL2-R $5^{\prime}$-TGGAGAAGTAGTACCACCACAA-3'; msPLA2-F $5^{\prime}$-CTGTCAGATGCACGACCGTTGT- $3^{\prime}$ and msPLA2-R $5^{\prime}$ - GAGCCTCATTGGACAGAAGGAG-3'; $\mathrm{m} \beta$-actin- $\mathrm{F} 5^{\prime}$ - ACCCTAAGGCCAACCGTGAA- $3^{\prime}$ and $\mathrm{m} \beta$-actin-R $5^{\prime}$ - ATGGCGTGAGGGAGAGCATAG- $3^{\prime}$.

\section{Statistical analysis}

The data are presented as the mean $\pm \mathrm{SD}$. All data were analyzed by one-way ANOVA followed by the Tukey's post hoc test for multiple comparisons using Prism 7.0 software (GraphPad). Survival rates were estimated by the Kaplan-Meier method and compared by the pairwise log-rank test. $p<0.05$ was considered statistically significant. PCA (principal component analysis) was performed by MetaboAnalyst 4.0 (https://www.metaboanalyst. ca) [18]. Hierarchical clustering analysis(heatmap) and metabolic pathway analysis (bubble plot) were performed by ImageGP (https://www.ehbio.com/ImageGP/).

\section{Results}

\section{PCTR1 improves the survival rate in an LPS-induced acute inflammatory mouse model}

Compared with the LPS group, the survival rate was significantly higher in the LPS + PCTR1 group, indicating that PCTR1 markedly increases the survival rate (Fig. 1a).

\section{PCTR1 ameliorates morphological damage of the lung, liver, and kidney in LPS-induced acute inflammation}

To identify when the acute inflammation is triggered in the LPS-induced acute inflammatory model, we investigated the effects of LPS on inflammatory factors (IL-1 $\beta$, IL-6, IL-10, and TNF- $\alpha$ ) over time. We found that the inflammatory factors were increased markedly at $1 \mathrm{~h}$ after LPS injection (Fig. 1b). Pathologic examination revealed that 


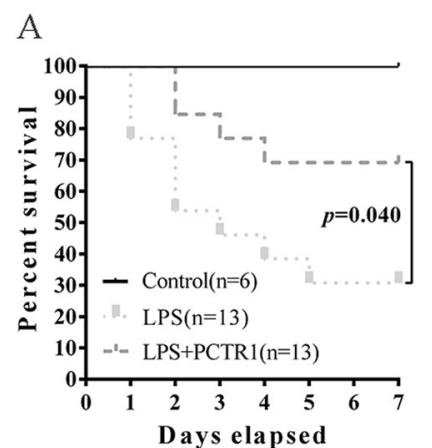

B

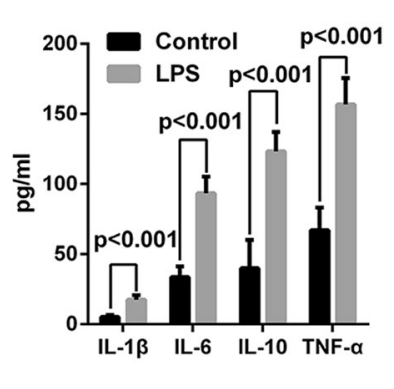

Fig. 1 PCTR1 improves the survival rate in the LPS-induced acute inflammatory mouse model. a PCTR $1(50 \mathrm{ng} /$ mouse $)$ was administered to the mice via the tail vein $1 \mathrm{~h}$ after LPS $(15 \mathrm{mg} / \mathrm{kg}$,) by intraperitoneal injection. And an equal volume of saline was given in both the control group and the LPS group. The survival rate of mice was recorded for 7 days. b The inflammatory factors IL-1 $\beta$, IL-6, IL-10, and TNF- $\alpha$, are increased markedly $1 \mathrm{~h}$ after LPS challenge. Data are expressed as mean \pm SD. $n=7$ per group.

the LPS group displayed significant damage of the lung, liver, and kidney, with interstitial lung edema, hemorrhage, and infiltration of inflammatory cells into the interstitium and alveolar spaces (Fig. 2a), hepatocyte degeneration and necrosis in the hepatic lobules (Fig. 2c), inflammatory cells infiltration in renal interstitium, microthrombotic obstruction in the glomerulus and renal tubules (Fig. 2e). All morphologic changes observed in the LPS group were less pronounced in the LPS + PCTR1 group (Fig. 2a, c, Fig. 3e). Tissue injury scores were quantified in parallel with pathophysiological changes (Fig. 2b, d, f).

\section{PCTR1 reduces LPS-stimulated serum inflammatory factor levels}

The levels of IL-1 $\beta$ (Fig. 3a), IL-6 (Fig. 3b), IL-10 (Fig. 3c), TNF- $\alpha$ (Fig. 3d), MIP-2 (Fig. 3e), and MPO (Fig. 3f) were significantly higher in the LPS group than in the control group, and substantially lower in the PCTR1 treatment group. To further examine the effects of PCTR1 on the inflammatory factors, treatment of PCTR1 was delayed. The levels of inflammatory factors in the LPS + PCTR1 group were also lower than in the LPS group (Fig. 3g-1) when PCTR1 was given at $6 \mathrm{~h}$ after LPS challenge.

\section{PCTR1 ameliorates organs function in LPS-induced acute inflammation}

The $\mathrm{PaO}_{2}$ (arterial partial pressure of oxygen) levels (Fig. 4a) were higher and the $\mathrm{PaCO}_{2}$ (arterial partial pressure of carbon dioxide) levels (Fig. 4b) were lower in the
LPS + PCTR1 group compared with that in the LPS group. In addition, the liver function biomarkers ALT (Fig. 4c) and AST (Fig. 4d), and the kidney function biomarkers BUN (Fig. 4e) and CREA (Fig. 4f), were significantly reduced after PCTR1 treatment.

\section{PCTR1 decreases LPS-stimulated serum LA and AA levels detected by metabolomics analysis and HPLC- MS}

To explore the specific mechanisms of the organ protection effects of PCTR1, metabolomics analysis was performed. Principal component analysis (PCA) showed that there were distinct differences in metabolites between groups (Fig. 5a, control vs. LPS; Fig. 5d, LPS vs. LPS + PCTR1). Heatmaps of hierarchical clustering analysis were conducted to show the differentially expressed metabolites for control vs. LPS or LPS vs. LPS + PCTR1 (Fig. 5b, e). The serum concentrations of LA and AA were significantly higher in the LPS group than in the control group (marked with red, Fig. 5b), while the changes were reversed after PCTR1 treatment (marked with red, Fig. 5e). Bubble plots of metabolic pathway analysis showed that LA metabolism was the most highly influenced pathway after LPS administration and PCTR1 treatment (both impact index $=1$, the maximum; Fig. 5c, f). The relative quantities of LA and AA showed that PCTR1 decreased the LPS-stimulated serum LA and AA levels (Fig. 5g, h). The results were verified by HPLC-MS with the same samples (Fig. 5i, j).

\section{PCTR1 upregulates FADS1, FADS2, and ELOVL2 expression through activation of ALX to promote the conversion of LA to $A A$ in liver}

The mRNA expression of FADS1 (Fig. 6a), FADS2 (Fig. 6b), and ELOVL2 (Fig. 6c) in the liver tissues were significantly lower in the LPS group than in the control group; however, PCTR1 treatment promoted the expression of FADS1, FADS2, and ELOVL2 in a dose-dependent manner (Fig. 6a, b, c). To further illustrate the mechanism of PCTR1, the ALX antagonist (BOC-2) was used. We found that the effects of PCTR1 on FADS1 (Fig. 6d), FADS2 (Fig. 6e), and ELOVL2 (Fig. 6f) were partially blocked by BOC-2.

\section{PCTR1 downregulates LPS-stimulated PLA2 expression through activation of ALX to inhibit the conversion of bound $A A$ into free $A A$}

The mRNA expression and protein content of PLA2 in liver tissue were markedly increased in the LPS group 


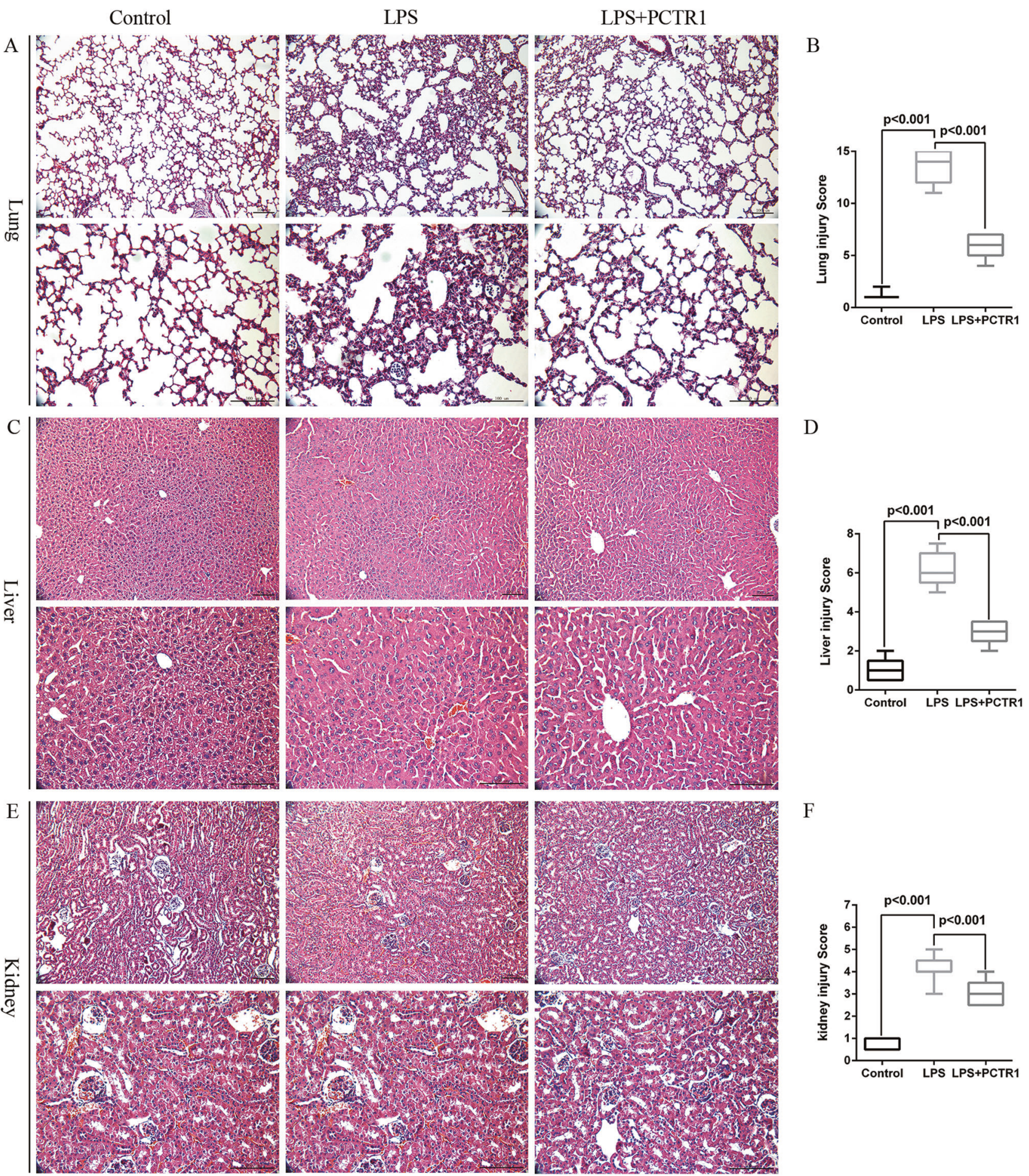

Fig. 2 PCTR1 ameliorates lung, liver, and kidney morphological damage. Lung, liver, and kidney tissues were obtained at $6 \mathrm{~h}$ after LPS injection. Representative images of lung (a), liver (c), e H\&E-stained sections (upper magnification $\times 200$; lower magnification $\times 400$ ) for the control, LPS, and LPS + PCTR1 groups, indicating that PCTR1 treatment strongly ameliorates LPS-induced lung edema, hemorrhage, and alveolar collapse (a), alleviates hepatocyte degeneration and necrosis in hepatic lobules (c), decreases inflammatory cells infiltration in the renal interstitium, microthrombotic obstruction in the glomerulus and renal tubules (e). Acute lung injury score (b), acute liver injury score (d), acute and kidney injury score (f). Data are presented as box plots. $n=7$ mice per group. 

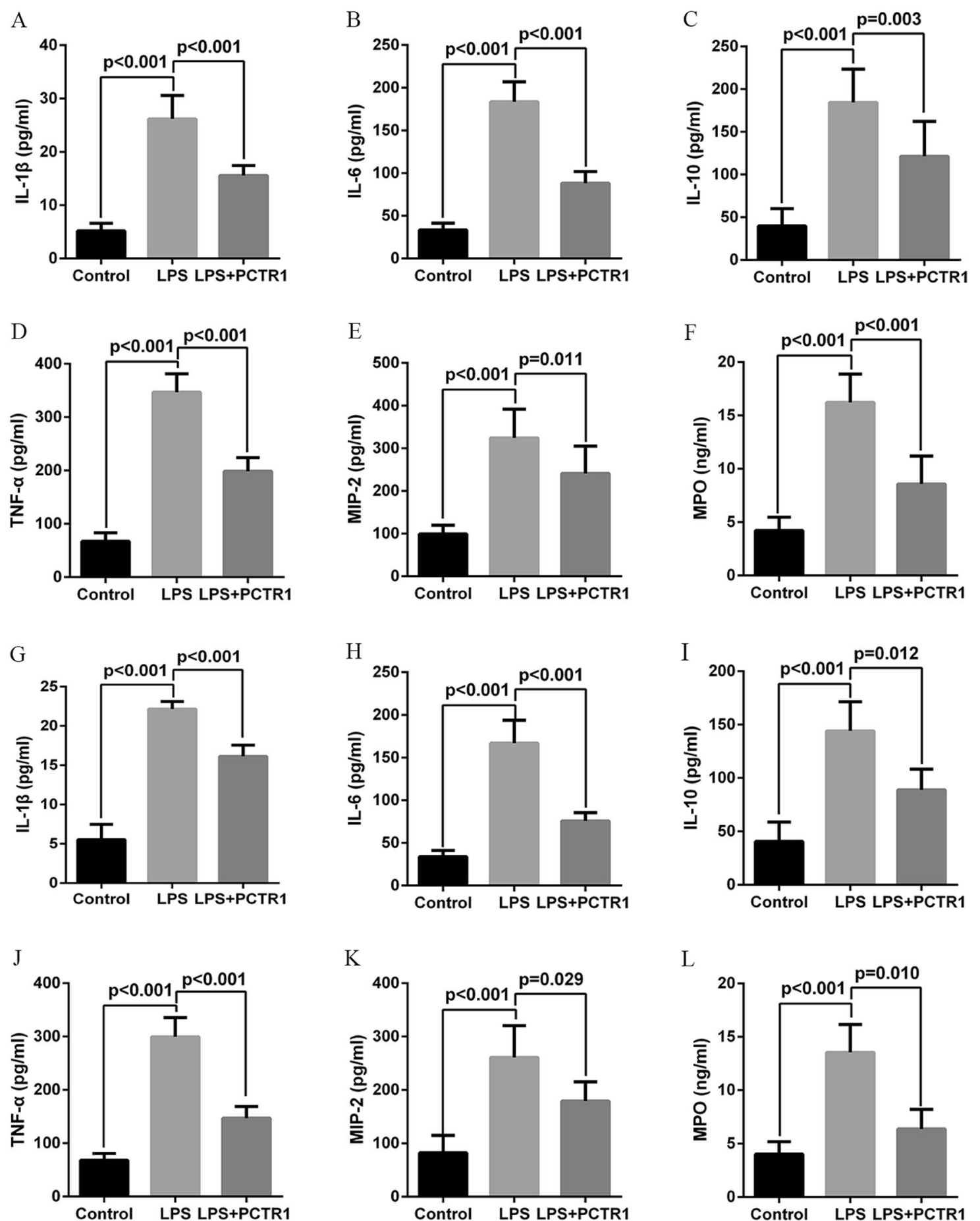

Fig. 3 PCTR1 reduces LPS-stimulated inflammation. PCTR1 treatment began $1 \mathrm{~h}$ after LPS administration, and serum levels of the inflammatory factors IL-1 $\beta$ (a), IL-6 (b), IL-10 (c), TNF- $\alpha$ (d), MIP-2 (e), and MPO (f), decreased significantly. Similarly, when PCTR1

treatment was delayed to $6 \mathrm{~h}$ after the LPS challenge, the inflammatory factors were inhibited as well $(\mathbf{g}-\mathbf{l})$. Data are shown as mean \pm SD. $n=7$ per group from two distinct experiments.

compared with the control group; however, PCTR1 treatment reduced the expression of PLA2 in a dose-dependent manner (Fig. 6g, h). There was no significant difference between the LPS group and the LPS + PCTR1 + BOC-2 group, showing that the effect of PCTR1 on PLA2 could be partially blocked by BOC-2; hence, PCTR1 might downregulate LPS-stimulated PLA2 expression through the activation of ALX (Fig. 6i, j). 

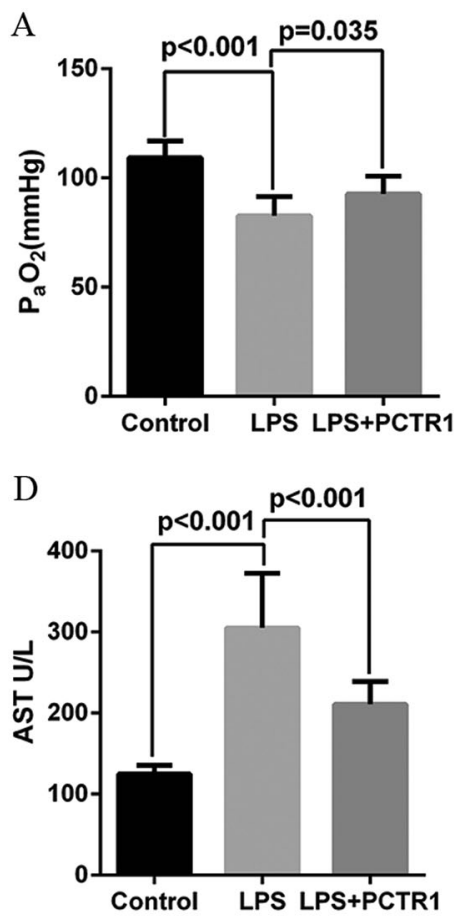

Fig. 4 PCTR1 ameliorates respiratory function, liver function, and kidney function. $\mathrm{PaO}_{2}$ was increased (a), while the $\mathrm{PaCO}_{2}$ was decreased (b) after PCTR1 treatment. The serum levels of ALT (c),
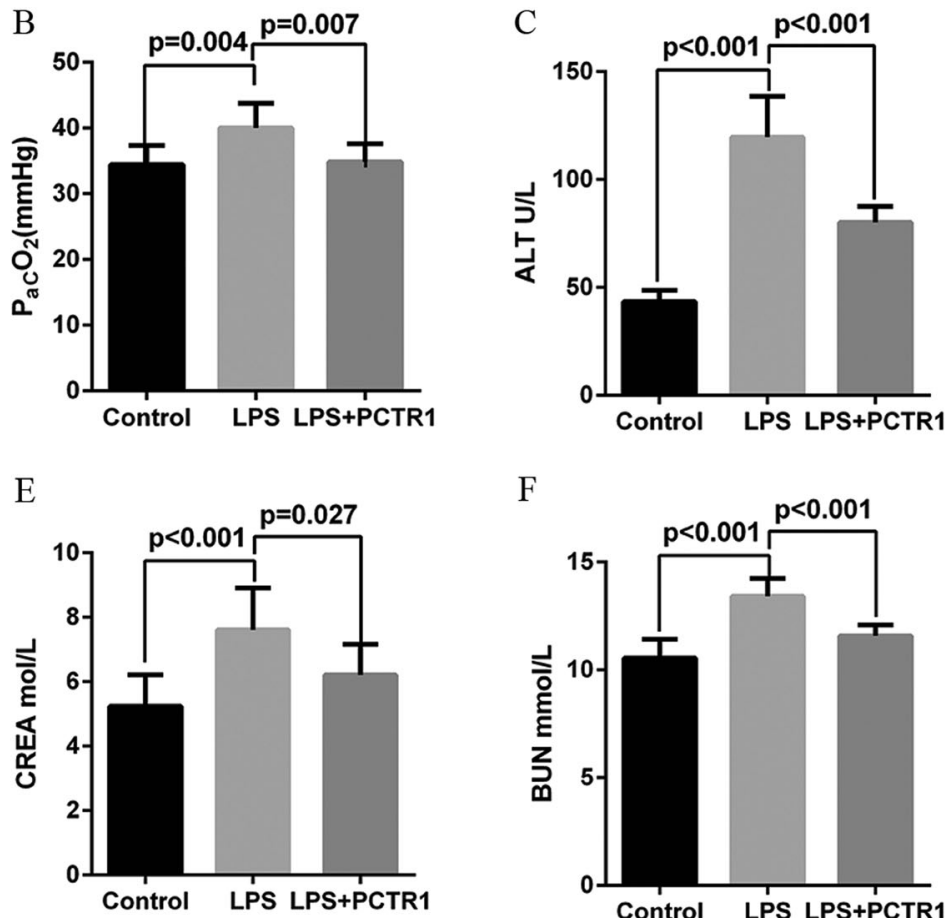

AST (d), BUN (e), and CREA (f) were significantly reversed by PCTR1 treatment. Data are expressed as mean \pm SD. $n=7$ per group from two distinct experiments.

\section{PCTR1 increases bound AA content in the liver and reduces free $A A$ levels in serum}

The AA levels in liver were significantly lower in the LPS group than in the control group, while PCTR1 treatment could reverse this trend (Fig. 6k). However, serum AA levels were markedly higher in the LPS group than in the control group, but lower in the LPS + PCTR1 group than in the LPS group (Fig. 5h, j).

\section{PCTR1 increases serum SOD and GPX4 levels and reduces serum PGE2 and ROS levels}

PGE2 is the major metabolite of AA and play a strongly proinflammatory effect [19]. The serum PGE2 (Fig. 7a) and ROS (Fig. 7b) levels were higher and levels of serum SOD (Fig. 7c) and GPX4 (Fig. 7d) were lower in the LPS group compared with those in the control group. These effects of LPS were markedly reversed after PCTR1 treatment.

\section{Discussion}

In the present study, we confirmed that PCTR 1 could effectively ameliorate acute inflammation and morphological damage and dysfunction of the lung, liver, and kidney, as well as improve the survival rate in LPS-induced acute inflammatory mouse model. Metabolomics analysis showed that LA metabolism was the most highly influenced metabolite after PCTR1 treatment. In addition, PCTR1 reduced LPS-stimulated serum LA, AA, and PGE2 levels. PCTR1, via the activation of ALX, promotes the conversion of LA to AA by increasing the expression of FADS1, FADS2, and ELOVL2 in the liver, and inhibits the conversion of bound AA into free AA by reducing the expression of PLA2 so as to decrease the serum AA and PGE2 levels (summarized in Fig. 8).

Dalli $\mathbf{J}$ et al. demonstrated that PCTR1 could rectify peritoneal macrophage and host responses to $E$. coli infection in vagotomized mice [7]. Similarly, Ramon $S$ et al. reported that PCTR1 enhanced peritoneal macrophage recruitment and phagocytosis of E. coli, and decreased polymorphonuclear leukocyte infiltration [8]. Our study also shows that PCTR1 could reduce LPS-induced infiltration of inflammatory cells in the lung, liver, and kidney. Moreover, we found that PCTR1 could ameliorate respiratory function, liver function, and kidney function and increase the survival rate of LPS-induced acute inflammatory mice, indicating that PCTR 1 has beneficial effects against inflammation.

Metabolomics analysis was applied to investigate the metabolic pathways altered by PCTR1 treatment, and we 
A

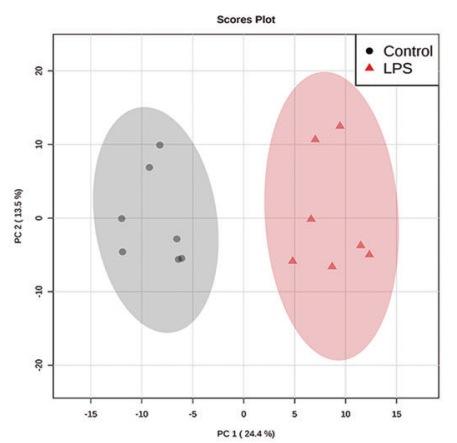

C

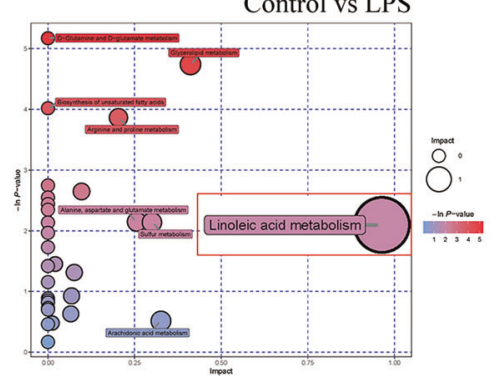

D

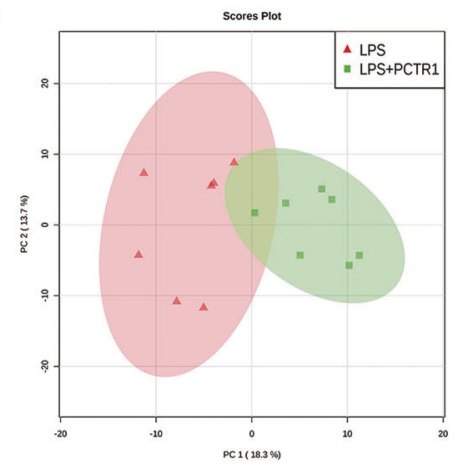

F

LPS vs LPS+PCTR1

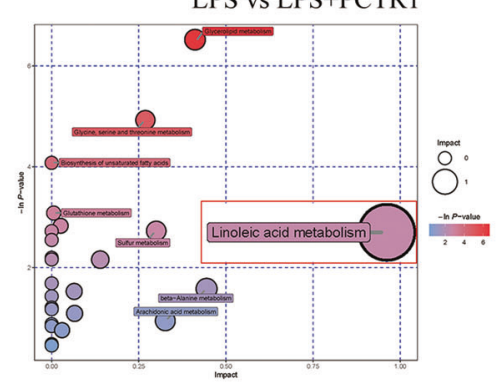

E
B
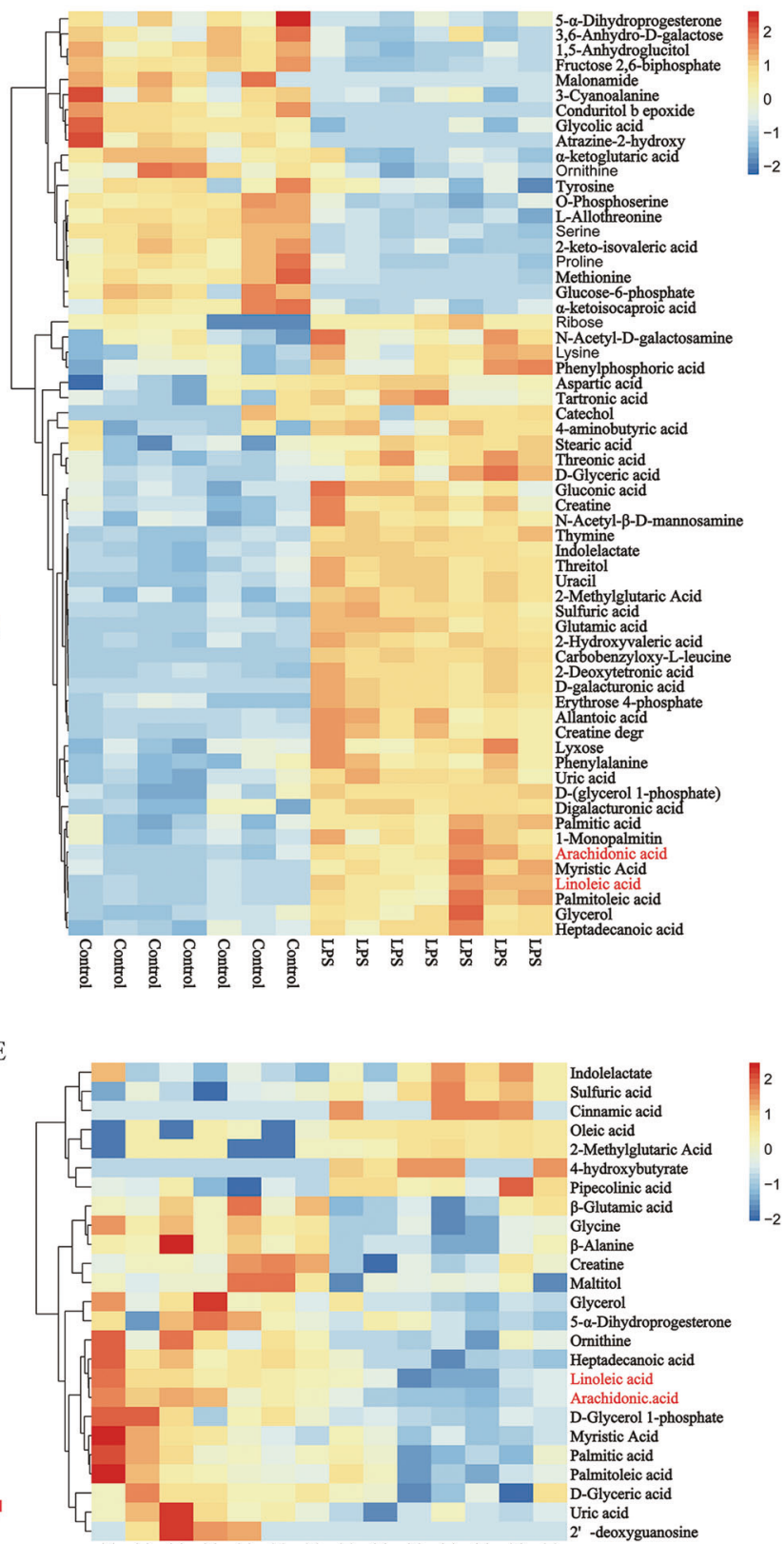

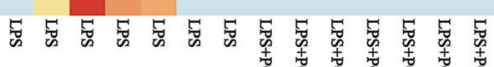

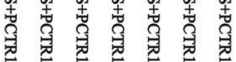
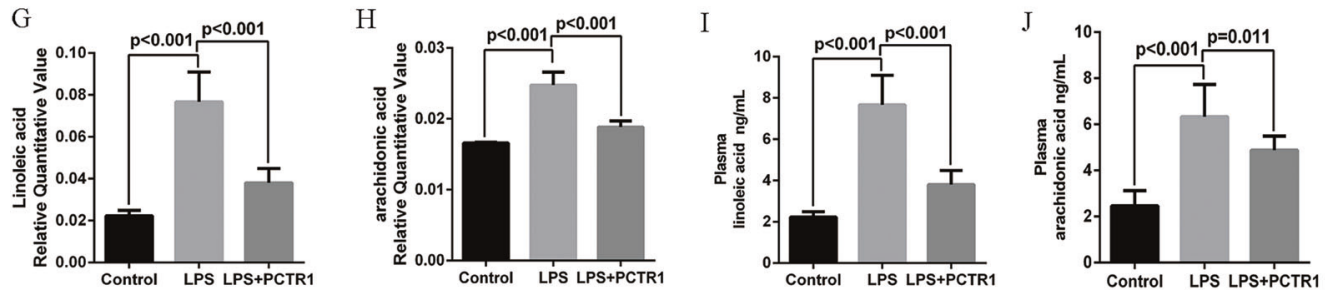
Fig. 5 PCTR1 decreases LPS-stimulated serum LA and AA levels. Metabolomics analysis was performed by GC-TOF-MS (see MATERIALS AND METHODS for details). 2D scores scatter plots of PCA model for control vs. LPS and LPS vs. LPS + PCTR1, all samples are within 95\% confidence regions (a, d). Heatmap of hierarchical clustering analysis for differentially expressed metabolites, control vs. LPS (b), LPS vs. LPS + PCTR1 (e). Bubble plot for pathway analysis, the LA metabolism is the most greatly influenced pathway (impact index $=1$, marked with a red border), control vs. LPS (c), LPS vs. LPS + PCTR1 (f). The relative quantitative values of LA and AA of the three groups $(\mathbf{g}, \mathbf{h})$. The absolute quantitative values of serum LA and AA were measured by HPLC-MS $(\mathbf{i}, \mathbf{j})$. Error bars represent mean \pm SD, $n=7$ per group.

found that LA metabolism was most affected. In addition, both metabolomics analysis and HPLC-MS analysis showed that LPS significantly increases the serum LA levels and AA levels. As AA is metabolized into pro-inflammatory cytokines, such as PGE2, TXA2, and LTB4 [19], the increased serum LA and AA might play an important role in LPS-induced acute inflammation [20]. However, PCTR1 treatment influenced LA metabolism, and attenuate LPSstimulated serum LA, AA, and PGE2 levels markedly, indicating that PCTR1 might display potential for organ protection and an anti-inflammatory role by reducing serum AA and PGE2 levels.

LA is formed from essential fatty acids by desaturases, including FADS1, FADS2, and ELOVL2 [21]. LA is converted into GLA ( $\gamma$-linolenic acid) by FADS2, and GLA is converted into DGLA (dihomo- $\gamma$-linolenic acid) with the catalyzation of FADS1 and ELOVL2. Finally, DGLA is converted to AA [3]. Previous research has shown that the expression and the activity of desaturases are suppressed by LPS and cytokines, such as IL- 6 and TNF- $\alpha$, induced by LPS [3, 20-22]. Our study also confirmed that the expression of desaturases (FADS1, FADS2, ELOVL2) are reduced by LPS in the liver tissue, indicating that LPS stimulation might increase serum LA levels through inhibition of the conversion of LA to AA by downregulating the expression of desaturases. Contrariwise, PCTR1 upregulates the expression of desaturases to promote the conversion of LA to AA, leading to reducing serum LA levels and increasing the intrahepatic AA content. Consistent with our results, vitamin $\mathrm{C}$, folic acid and vitamin B6 helped maintain normal activity of desaturases [20], implying that PCTR1 might have a similar effect as these vitamins.

AA is present in the phospholipids of cellular membranes to maintain cytomembrane integrity and function [3, 4]. However, with the catalyzation of PLA2, the bound AA is hydrolyzed into free AA in the circulation, resulting in proinflammatory cytokines storm [3, 4]. Furthermore, when LPS insults the lung, the liver tissue, and liver macrophages, the expression and enzyme activity of PLA2 will be accelerated leading to inflammatory responses and organ damage [23, 24]. PLA2 is also recognized as a mediator in septic shock [25]. In our study, we demonstrated that LPS increases the expression of intrahepatic PLA2 in mRNA level and protein level, as well as the serum levels of AA and PGE2. Treatment with PCTR1, however, these trends are reversed, indicating that PCTR1 might inhibit the conversion of bound AA into free AA by decreasing the expression of intrahepatic PLA2 to reduce the serum LA and PGE2 level. Several researchers have demonstrated that the pro-inflammatory bioactive lipids could be switched to anti-inflammatory in sepsis patients and animal model [26, 27]. Our previous research has illustrated that SPM, such as RvD1, could promote this process by affect cyclooxygenase-2 expression [26], implying that PCTR1 may also have an effect on this process to reduce inflammation.

The specific receptor for PCTR1 is unknown. Previous research has shown that the functions of SPMs, such as resolvin D1, maresin1, and protectin DX, were partially abolished by a ALX antagonist (BOC-2) [7, 11, 12]. In this study, effects of PCTR1 on desaturases and PLA2, and intrahepatic AA content were also partially inhibited by BOC-2. Collectively, these results suggest that the properties of PCTR1 might be ALX dependent.

Several SPMs, such as resolvin D1, resolvin D5, resolvin E1, were correlated with high mortality in patients with sepsis, implying that SPMs might be potential clinical biomarkers for predicting survival [28, 29]. We previously found that the levels of resolvins, protectins, and maresins were lower in nonsurvivors of sepsis than those in survivors, but the values did not achieve a statistically significant level. It is noteworthy that any one metabolite may not make much difference by itself, but when these antiinflammatory compounds are grouped together their effect may be significant [28]. Accordingly, more samples will be processed in the near future. Because lipid metabolism is intricate, especially in inflammation, all molecules need to be considered in terms of their coordinated actions [28]. The balance between ROS and GPX4 exerts an important role in the inflammation, and SOD is a regulator of this balance [26]. In this study, the organ protection and antiinflammation effects of PCTR1 depend on its combined actions of increasing SOD and GPX4 and reducing inflammatory factors such as PGE2 and ROS.

DHA released from the cell membrane undergoes lipoxygenation, followed by epoxidation and hydrolysis to generate protectin, while PCTR1 is derived from protectin [27, 28, 30-32]. The metabolites of DHA, such as protectin, resolvin and maresin, intervene the activity of desaturases through BDNF to affect the metabolism of AA [20, 27]. On the contrary, the metabolites of AA also cross-talk with 
A

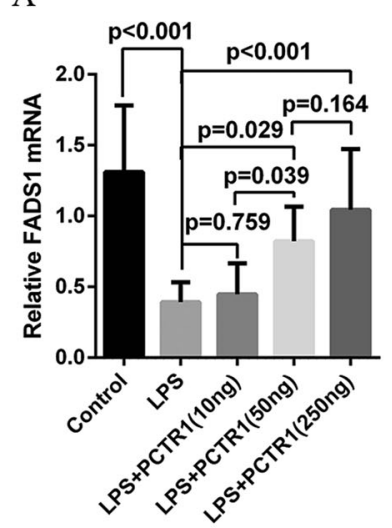

$\mathrm{E}$

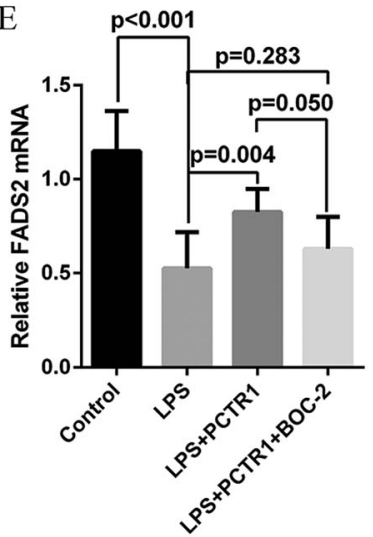

I

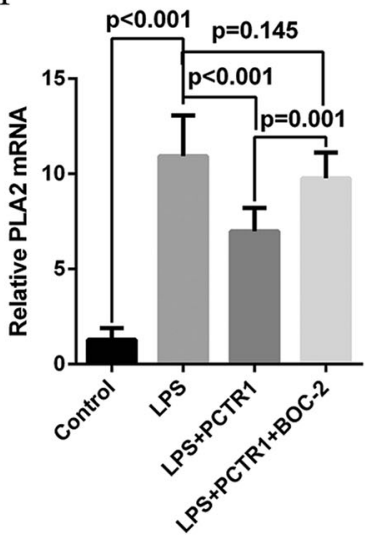

B

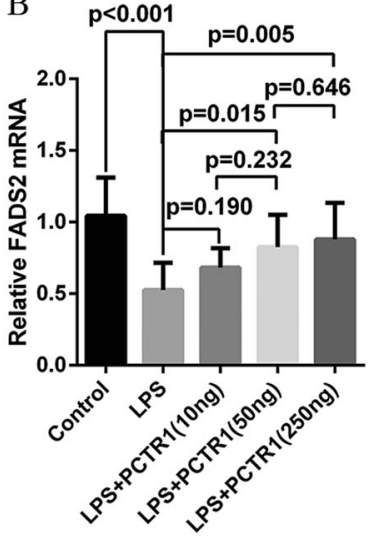

F

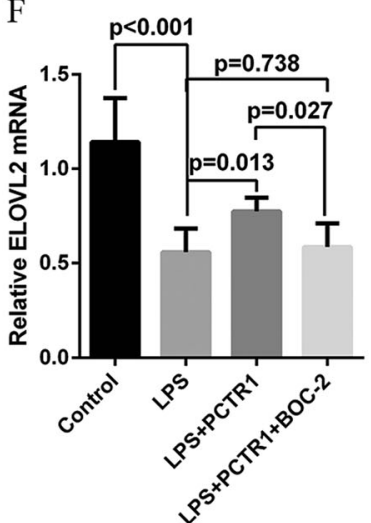

$\mathrm{J}$

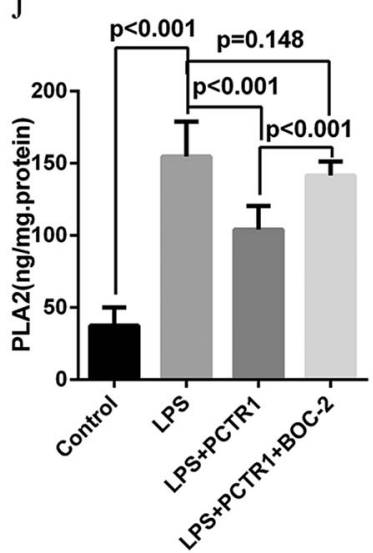

$\mathrm{C}$

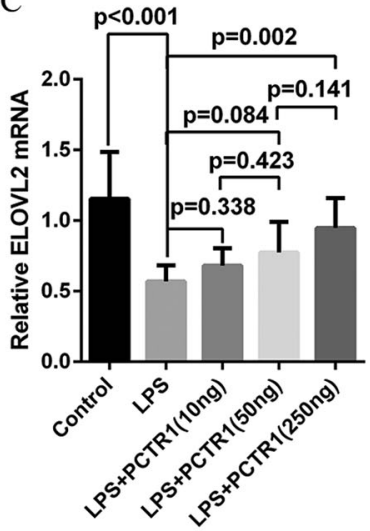

G

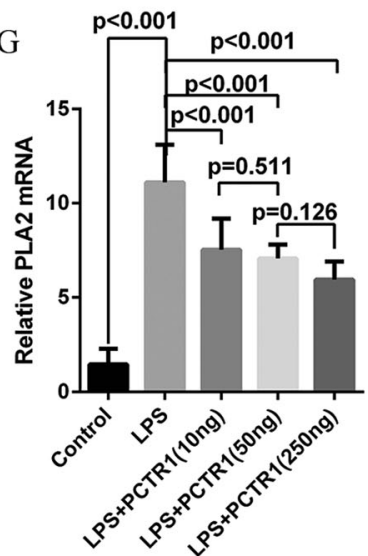

K

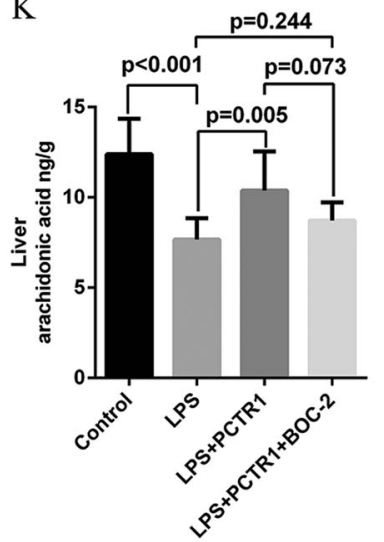

$\mathrm{D}$
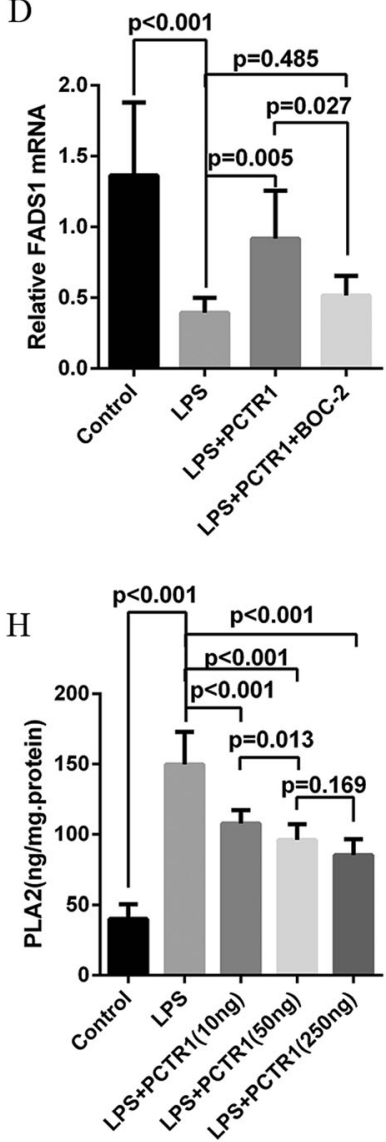

Fig. 6 PCTR1 regulates the key enzymes of LA metabolism. mRNA expression of FADS1, FASDS2, ELOV2, and PLA2 in the liver tissue $(\mathbf{a}-\mathbf{g}, \mathbf{i})$. The protein expression of PLA2 in the liver tissue

DHA through desaturases and BDNF [20, 27]. The effects of fatty acids/AA/DHA and their metabolites on inflammation await further investigation.

Taken together, our results demonstrate that PCTR1, through the activation of ALX, promotes the conversion of LA to AA by increasing the expression of FADS1, FASDS2, and ELOV2, and inhibits the conversion of bound

homogenate, performed by ELISA kits $(\mathbf{h}, \mathbf{j})$. Intrahepatic AA content was detected by HPLC-MC (k). Error bars represent mean $\pm \mathrm{SD}, n=7$ per group from two distinct experiments $(\mathbf{a}-\mathbf{j}), n=7$ per group $(\mathbf{k})$.
AA into free AA by downregulating the expression of PLA2, leading to reduction of the serum AA and PGE2 levels. Subsequently, PCTR1 treatment decreases inflammation and ameliorates organ damage and dysfunction, thereby improving the survival of mice with LPS-induced acute inflammation. Our findings have revealed a novel mechanism for anti-inflammation of PCTR1 and suggest 
A

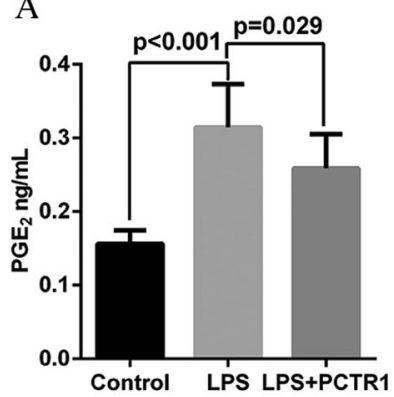

B

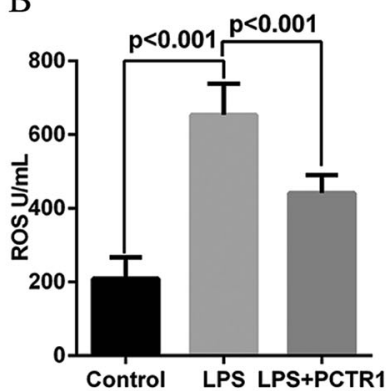

$\mathrm{C}$

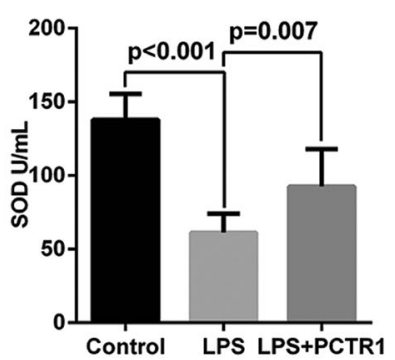

$\mathrm{D}$

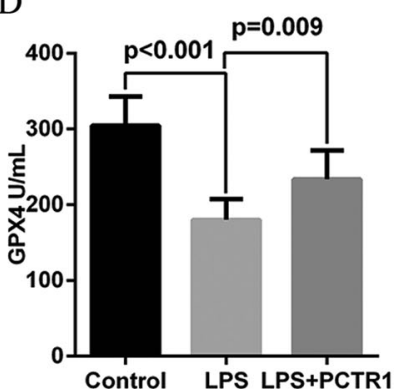

Fig. 7 PCTR1 increases serum SOD and GPX4 levels and reduces serum PGE2 and ROS levels. Serum PGE2 (a), ROS (b), SOD (c), GPX4 (d) levels were detected by ELISA. Data are expressed as mean \pm SD. $n=7$ per group.

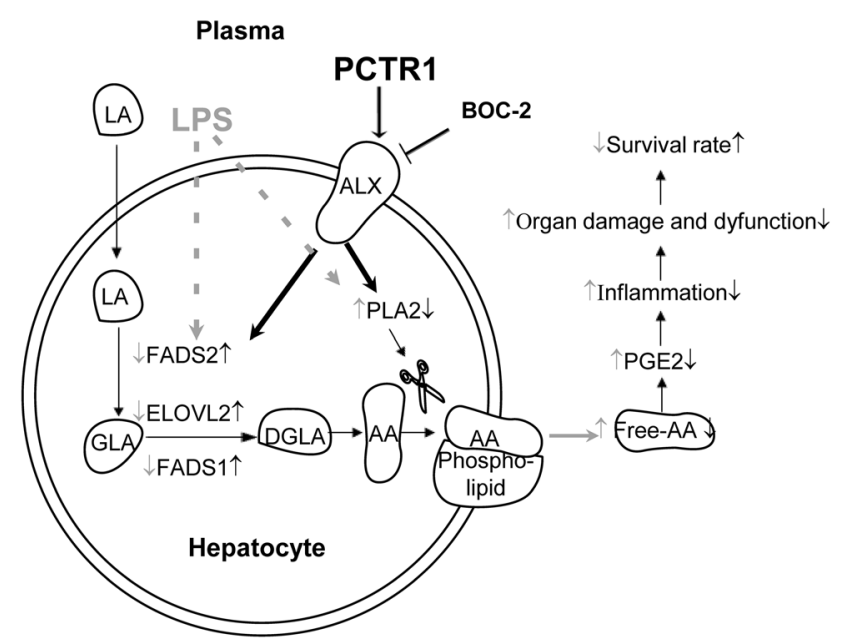

Fig. 8 Schematic mechanism of PCTR1 regulates LA metabolism by promoting FADS1/FASDS2/ELOV2 expression and inhibiting PLA2 expression. LPS lipopolysaccharide, LA linoleic acid, GLA $\gamma-$ linolenic acid, DGLA dihomo- $\gamma$-linolenic acid, AA arachidonic acid, FADS1 fatty acid desaturase 1, FADS2 fatty acid desaturase 2, ELOVL2 elongase of very long chain fatty acids, PLA2 phospholipase A2, PCTR1 protectin conjugates in tissue regeneration 1, ALX lipoxin A4 receptor, BOC-2 ALX antagonist. Gray arrows indicate the effects of LPS; black arrows indicate the effects of PCTR1.

that PCTR1 is a promising potential therapeutic target for acute inflammation.

Acknowledgements We thank Jian-Guang Wang, Qian Wang, and Hong-Xia Mei for their supports and advices in our experiments. We are grateful to Biotree Bio-technology Co., Ltd. (Shanghai, China) for providing helps in data analysis. This work was funded by the grants from the National Natural Science Foundation of China (No. 81571862, No. 81870065), Natural Science Foundation of Zhejiang Province (No. LQ20H150003, No. LY19H150002), and Wenzhou Municipal Science and Technology Bureau (no. Y20190087, no. Y20190118).

\section{Compliance with ethical standards}

Conflict of interest The authors declare that they have no conflict of interest.
Publisher's note Springer Nature remains neutral with regard to jurisdictional claims in published maps and institutional affiliations.

\section{References}

1. Simpson BW, Trent MS. Pushing the envelope: LPS modifications and their consequences. Nat Rev Microbiol. 2019; 17:403-16.

2. Bannerman DD, Goldblum SE. Mechanisms of bacterial lipopolysaccharide-induced endothelial apoptosis. Am J Physiol Lung Cell Mol Physiol. 2003;284:L899-914.

3. O'Neill CM, Minihane AM. The impact of fatty acid desaturase genotype on fatty acid status and cardiovascular health in adults. Proc Nutr Soc. 2017;76:64-75.

4. Hishikawa D, Shindou H, Kobayashi S, Nakanishi H, Taguchi R, Shimizu T. Discovery of a lysophospholipid acyltransferase family essential for membrane asymmetry and diversity. Proc Natl Acad Sci U S A. 2008;105:2830-5.

5. Rinschen MM, Ivanisevic J, Giera M, Siuzdak G. Identification of bioactive metabolites using activity metabolomics. Nat Rev Mol Cell Biol. 2019;20:353-67.

6. Hao Y, Zheng H, Wang RH, Li H, Yang LL, Bhandari S, et al. Maresin1 alleviates metabolic dysfunction in septic mice: A (1)H NMR-based metabolomics analysis. Mediators Inflamm. 2019;2019:2309175.

7. Dalli J, Colas RA, Arnardottir H, Serhan CN. Vagal regulation of group 3 innate lymphoid cells and the immunoresolvent PCTR1 controls infection resolution. Immunity. 2017;46:92-105.

8. Ramon S, Dalli J, Sanger JM, Winkler JW, Aursnes M, Tungen JE, et al. The protectin PCTR1 is produced by human M2 macrophages and enhances resolution of infectious inflammation. Am J Pathol. 2016;186:962-73.

9. Samuelsson B. Role of basic science in the development of new medicines: examples from the eicosanoid field. J Biol Chem. 2012;287:10070-80.

10. Baillie JK, Digard P. Influenza-time to target the host? N Engl J Med. 2013;369:191-3.

11. Yang JX, Li M, Chen XO, Lian QQ, Wang Q, Gao F, et al. Lipoxin A4 ameliorates lipopolysaccharide-induced lung injury through stimulating epithelial proliferation, reducing epithelial cell apoptosis and inhibits epithelial-mesenchymal transition. Respir Res. 2019;20:192.

12. Zhang HW, Wang Q, Mei HX, Zheng SX, Ali AM, Wu QX, et al. RvD1 ameliorates LPS-induced acute lung injury via the suppression of neutrophil infiltration by reducing CXCL2 expression and release from resident alveolar macrophages. Int Immunopharmacol. 2019;76:105877. 
13. Zhuo XJ, Hao Y, Cao F, Yan SF, Li H, Wang Q, et al. Protectin DX increases alveolar fluid clearance in rats with lipopolysaccharide-induced acute lung injury. Exp Mol Med. 2018;50:49.

14. El-Lakany MA, Fouda MA, El-Gowelli HM, El-Gowilly SM, ElMas MM. Gonadal hormone receptors underlie the resistance of female rats to inflammatory and cardiovascular complications of endotoxemia. Eur J Pharmacol. 2018;823:41-48.

15. Dunn WB, Broadhurst D, Begley P, Zelena E, Francis-McIntyre $\mathrm{S}$, Anderson N, et al. Procedures for large-scale metabolic profiling of serum and plasma using gas chromatography and liquid chromatography coupled to mass spectrometry. Nat Protoc. 2011;6:1060-83.

16. Lin X, Zhao L, Tang S, Zhou Q, Lin Q, Li X, et al. Metabolic effects of basic fibroblast growth factor in streptozotocin-induced diabetic rats: A (1)H NMR-based metabolomics investigation. Sci Rep. 2016;6:36474.

17. Kind T, Wohlgemuth G, Lee DY, Lu Y, Palazoglu M, Shahbaz S, et al. FiehnLib: mass spectral and retention index libraries for metabolomics based on quadrupole and time-of-flight gas chromatography/mass spectrometry. Anal Chem. 2009;81:10038-48.

18. Chong J, Wishart DS, Xia J. Using MetaboAnalyst 4.0 for comprehensive and integrative metabolomics data analysis. Curr Protoc Bioinformatics. 2019;68:e86.

19. Alhouayek M, Muccioli GG. COX-2-derived endocannabinoid metabolites as novel inflammatory mediators. Trends Pharmacol Sci. 2014;35:284-92.

20. Das UN. Polyunsaturated fatty acids and sepsis. Nutrition. 2019;65:39-43.

21. Castro LF, Tocher DR, Monroig O. Long-chain polyunsaturated fatty acid biosynthesis in chordates: insights into the evolution of Fads and Elovl gene repertoire. Prog Lipid Res. 2016;62:25-40.

22. Gu M, Li Y, Tang H, Zhang C, Li W, Zhang Y, et al. Endogenous omega (n)-3 fatty acids in Fat-1 mice attenuated depression-like behavior, imbalance between microglial M1 and M2 phenotypes, and dysfunction of neurotrophins induced by lipopolysaccharide administration. Nutrients. 2018;10:1351.

23. Rozenfeld RA, Liu X, DePlaen I, Hsueh W. Role of gut flora on intestinal group II phospholipase A2 activity and intestinal injury in shock. Am J Physiol Gastrointest Liver Physiol. 2001;281: G957-963.

24. Dieter P, Kolada A, Kamionka S, Schadow A, Kaszkin M. Lipopolysaccharide-induced release of arachidonic acid and prostaglandins in liver macrophages: regulation by Group IV cytosolic phospholipase A2, but not by Group V and Group IIA secretory phospholipase A2. Cell Signal. 2002;14:199-204.

25. Scott DL, White SP, Browning JL, Rosa JJ, Gelb MH, Sigler PB. Structures of free and inhibited human secretory phospholipase A2 from inflammatory exudate. Science. 1991;254:1007-10.

26. Gao Y, Zhang H, Luo L, Lin J, Li D, Zheng S, et al. Resolvin D1 improves the resolution of inflammation via activating NF-kappaB p50/p50-mediated cyclooxygenase- 2 expression in acute respiratory distress syndrome. J Immunol. 2017;199:2043-54.

27. Das UN. n-3 fatty acids, $\gamma$-linolenic acid, and antioxidants in sepsis. Crit Care. 2013;17:312.

28. Das UN. Combination of aspirin with essential fatty acids is superior to aspirin alone to prevent or ameliorate sepsis or ARDS. Lipids Health Dis. 2016;15:206.

29. Dalli J, Colas RA, Quintana C, Barragan-Bradford D, Hurwitz S, Levy BD, et al. Human sepsis eicosanoid and proresolving lipid mediator temporal profiles: correlations with survival and clinical outcomes. Crit Care Med. 2017;45:58-68.

30. Das UN. Is sepsis a pro-resolution deficiency disorder? Med Hypotheses. 2013;80:297-9.

31. Das UN. HLA-DR expression, cytokines and bioactive lipids in sepsis. Arch Med Sci: AMS. 2014;10:325-35.

32. Das UN. Circulating microparticles in septic shock and sepsisrelated complications. Minerva Anestesiol. 2019;85:571-6. 